\title{
Chiral Fermions and Quadratic Divergences
}

\author{
Xavier Calmet, Paul H. Frampton and Ryan M. Rohm \\ University of North Carolina, Chapel Hill, NC 27599-3255, USA.
}

\begin{abstract}
In an approach towards naturalness without supersymmetry, the renormalization properties of nonsupersymmetric abelian quiver gauge theories are studied. In the construction based on cyclic groups $Z_{p}$ the gauge group is $U(N)^{p}$, the fermions are all in bifundamentals and the construction allows scalars in adjoints and bifundamentals. Only models without adjoint scalars, however, exhibit both chiral fermions and the absence of one-loop quadratic divergences in the scalar propagator.
\end{abstract}

Typeset using REVTEX 


\section{Introduction}

One of the principal motivations for extending the standard model of particle phenomenology, over the last three decades and more, has been the concern about naturalness of the light Higgs scalar mass (e.g. [1]). One expects new physics to appear well above the weak scale, for example at the Planck scale for gravity, at a GUT scale for grand unification or at a right-handed neutrino mass scale for the see-saw mechanism of neutrino mass. In any such case the appearance of quadratic divergences in the Higgs scalar propagator of the standard model would suggest a heavy Higgs scalar mass thus necessitating fine-tuning and unnaturalness.

There are several popular approaches to this naturalness question; here we shall discuss a less popular but seemingly equally valid approach. The popular ideas are (i) that the scalar is a bound state analogous to a Cooper pair in BCS theory [2], though no fully convincing model exists; (ii) that there is an extra symmetry, supersymmetry [3], which protects the light Higgs mass once it is introduced by hand; (iii) that there are large extra dimensions [4] near the weak scale which avoid the need of a much higher physics scale; (iv) that among the $\sim 10^{100}$ vacua of string theory the smallness $\sim 100 \mathrm{GeV}$ of the Higgs mass is correlated to the smaller scale $\sim 1 \mathrm{meV}$ of cosmic dark energy [5].

An alternative approach is to use nonsupersymmetric gauge theories derived from the most highly supersymmetric $\mathcal{N}=4$ gauge theories. Such nonsupersymmetric $\mathcal{N}=0$ gauge theories can be systematically constructed [6-9] from $\mathcal{N}=4$ ones by using suitable orbifolding. The resulting theories are not coupled to gravity; we here assume this vanishing-gravity limit to be a sufficiently accurate approximation to the physics of any foreseeable collider experiment, which would be sensitive only to non-gravitational interactions.

Models can be constructed with four-dimensional conformal invariance at high energies; for the models we consider here, the renormalization group $\beta$-functions vanish for all $\mathrm{SU}(\mathrm{N})$ gauge groups. However, other desirable properties require $U(N)$ gauge groups so there is still a subtlety of decoupling U(1) factors (discussed later).

For example, in [8] there is a $Z_{7}$ model which contains all the states of the standard 
model and in [9] there is a $Z_{12}$ model allowing grand unification at a scale $4 \mathrm{TeV}$. In the sequence of $Z_{p}$ models as we shall see the first with chiral fermions is $Z_{4}$ but the $Z_{7}$ and $Z_{12}$ examples also fall into the class we shall investigate. We shall then discuss the quadratic divergence of the scalar propagator at one loop.

\section{Classification of abelian quiver gauge theories}

We consider the compactification of the type-IIB superstring on the orbifold $A d S_{5} \times S^{5} / \Gamma$ where $\Gamma$ is an abelian group $\Gamma=Z_{p}$ of order $p$ with elements $\exp (2 \pi i A / p), 0 \leq A \leq(p-1)$.

The resultant quiver gauge theory has $\mathcal{N}$ residual supersymmetries with $\mathcal{N}=2,1,0$ depending on the details of the embedding of $\Gamma$ in the $S U(4)$ group which is the isotropy of the $S^{5}$. This embedding is specified by the four integers $A_{m}, 1 \leq m \leq 4$ with

$$
\Sigma_{m} A_{m}=0(\operatorname{modp})
$$

which characterize the transformation of the components of the defining representation of $S U(4)$.

We are here interested in the non-supersymmetric case $\mathcal{N}=0$ which occurs if and only if all four $A_{m}$ are non-vanishing.

The gauge group is $U(N)^{p}$. The fermions are all in the bifundamental representations

$$
\sum_{m=1}^{m=4} \Sigma_{j=1}^{j=p}\left(N_{j}, \bar{N}_{j+A_{m}}\right)
$$

which are manifestly non-supersymmetric because no fermions are in adjoint representations of the gauge group. Scalars appear in representations

$$
\sum_{i=1}^{i=3} \Sigma_{j=1}^{i=p}\left(N_{j}, \bar{N}_{j \pm a_{i}}\right)
$$

in which the six integers $\left(a_{i},-a_{i}\right)$ characterize the transformation of the antisymmetric second-rank tensor representation of $S U(4)$. The $a_{i}$ are given by $a_{1}=\left(A_{2}+A_{3}\right), a_{2}=$ $\left(A_{3}+A_{1}\right), a_{3}=\left(A_{1}+A_{2}\right)$

It is possible for one or more of the $a_{i}$ to vanish in which case the corresponding scalar representation in the summation in Eq.(3) is to be interpreted as an adjoint representation 
of one particular $U(N)_{j}$. One may therefore have zero, two, four or all six of the scalar representations, in Eq.(3), in such adjoints. It is one purpose of the present article to investigate how the renormalization properties and occurrence of quadratic divergences depend on the distribution of scalars into bifundamental and adjoint representations.

Note that there is one model with all scalars in adjoints for each even value of $p$ (see Model Nos 1,3,12). For general even $p$ the embedding is $A_{m}=\left(\frac{p}{2}, \frac{p}{2}, \frac{p}{2}, \frac{p}{2}\right)$. This series is the complete list of $\mathcal{N}=0$ abelian quivers with all scalars in adjoints.

To be of more phenomenolgical interest the model should contain chiral fermions. This requires that the embedding be complex: $A_{m} \not \equiv-A_{m}(\bmod p)$. It will now be shown that for the presence of chiral fermions all scalars must be in bifundamentals.

The proof of this assertion follows by assuming the contrary, that there is at least one adjoint arising from, say, $a_{1}=0$. Therefore $A_{3}=-A_{2}(\bmod \mathrm{p})$. But then it follows from Eq.(1) that $A_{1}=-A_{4}(\bmod \mathrm{p})$. The fundamental representation of $S U(4)$ is thus real and fermions are non-chiral ${ }^{1}$.

The converse also holds: If all $a_{i} \neq 0$ then there are chiral fermions. This follows since by assumption $A_{1} \neq-A_{2}, A_{1} \neq-A_{3}, A_{1} \neq-A_{4}$. Therefore reality of the fundamental representation would require $A_{1} \equiv-A_{1}$ hence, since $A_{1} \neq 0, p$ is even and $A_{1} \equiv \frac{p}{2}$; but then the other $A_{m}$ cannot combine to give only vector-like fermions.

It follows that:

In an $\mathcal{N}=0$ quiver gauge theory, chiral fermions are possible

if and only if all scalars are in bifundamental representaions.

\footnotetext{
${ }^{1}$ This is almost obvious but for a complete justification, see [10]
} 
For the lowest few orders of the group $\Gamma$, the members of the infinite class of $\mathcal{N}=0$ abelian quiver gauge theories are tabulated below:

\begin{tabular}{|c|c|c|c|c|c|c|c|}
\hline Model No. & $\mathrm{p}$ & $A_{m}$ & $a_{i}$ & \begin{tabular}{|l|} 
scalar \\
bifunds.
\end{tabular} & $\begin{array}{l}\text { scalar } \\
\text { adjoints }\end{array}$ & $\begin{array}{c}\text { chiral } \\
\text { fermions? }\end{array}$ & $\begin{array}{l}\text { Contains } \\
\text { SM fields? }\end{array}$ \\
\hline 1 & $\mid 2$ & (1111) & $(000)$ & 0 & 6 & No & No \\
\hline 2 & 3 & (1122) & $(001)$ & 2 & 4 & No & No \\
\hline 3 & 4 & $(2222)$ & $(000)$ & 0 & 6 & No & No \\
\hline 4 & $\mid 4$ & (1133) & (002) & 2 & 4 & No & No \\
\hline 5 & $\mid 4$ & (1223) & (011) & 4 & 2 & No & No \\
\hline 6 & 4 & (1111) & $(222)$ & 6 & 0 & Yes & No \\
\hline 7 & $\mid 5$ & $(1144)$ & $(002)$ & 2 & 4 & No & No \\
\hline 8 & $\mid 5$ & $(2233)$ & (001) & 2 & 4 & No & No \\
\hline 9 & $\mid 5$ & (1234) & (012) & 4 & 2 & No & No \\
\hline 10 & $\mid 5$ & (1112) & (222) & 6 & 0 & Yes & No \\
\hline 11 & 5 & $(2224)$ & (111) & 6 & 0 & Yes & No \\
\hline 12 & 6 & (3333) & $(000)$ & 0 & 6 & No & No \\
\hline 13 & $\mid 6$ & (2244) & (002) & 2 & 4 & No & No \\
\hline 14 & 6 & $(1155)$ & $(002)$ & 2 & 4 & No & No \\
\hline 15 & 6 & $(1245)$ & $(013)$ & 4 & 2 & No & No \\
\hline 16 & $\mid 6$ & $(2334)$ & $(011)$ & 4 & 2 & No & No \\
\hline 17 & 6 & (1113) & $(222)$ & 6 & 0 & Yes & No \\
\hline 18 & $\mid 66$ & $(2235)$ & (112) & 6 & 0 & Yes & No \\
\hline 19 & $\mid 6$ & (1122) & (233) & 6 & 0 & Yes & No \\
\hline
\end{tabular}


The Table continues to infinity but we stop at $p=7$ :

\begin{tabular}{|c|c|c|c|c|c|c|c|}
\hline Model No. & $\mathrm{p}$ & $A_{m}$ & $a_{i}$ & $\begin{array}{l}\text { scalar } \\
\text { bifunds. }\end{array}$ & \begin{tabular}{|l|} 
scalar \\
adjoints
\end{tabular} & $\begin{array}{c}\text { chiral } \\
\text { fermions? }\end{array}$ & $\begin{array}{l}\text { Contains } \\
\text { SM fields? }\end{array}$ \\
\hline 20 & 7 & (1166) & $(002)$ & 2 & 4 & No & No \\
\hline 21 & 7 & $(3344)$ & (001) & 2 & 4 & No & No \\
\hline 22 & 7 & (1256) & (013) & 4 & 0 & No & No \\
\hline 23 & 7 & (1346) & (023) & 4 & 2 & No & No \\
\hline 24 & 7 & (1355) & (113) & 6 & 0 & No & No \\
\hline 25 & 7 & (1114) & (222) & 6 & 0 & Yes & No \\
\hline 26 & 7 & (1222) & (333) & 6 & 0 & Yes & No \\
\hline 27 & 7 & $(2444)$ & (111) & 6 & 0 & Yes & No \\
\hline 28 & 7 & (1123) & (233) & 6 & 0 & Yes & Yes \\
\hline 29 & 7 & $(1355)$ & (113) & 6 & 0 & Yes & Yes \\
\hline 30 & 7 & $(1445)$ & (122) & 6 & 0 & Yes & Yes \\
\hline
\end{tabular}




\section{Quadratic Divergences}

The lagrangian for the nonsupersymmetric $Z_{p}$ theory can be written in a convenient notation which accommodates simultaneously both adjoint and bifundamental scalars as

$$
\begin{aligned}
\mathcal{L}= & -\frac{1}{4} F_{\mu \nu ;, r, r}^{a b} F_{\mu \nu ; r, r}^{b a}+i \bar{\lambda}_{r+A_{4}, r}^{a b} \gamma^{\mu} D_{\mu} \lambda_{r, r+A_{4}}^{b a}+2 D_{\mu} \Phi_{r+a_{i}, r}^{a b \dagger} D_{\mu} \Phi_{r, r+a_{i}}^{b a}+i \bar{\Psi}_{r+A_{m}, r}^{a b} \gamma^{\mu} D_{\mu} \Psi_{r, r+A_{m}}^{b a} \\
& -2 i g\left[\bar{\Psi}_{r, r+A_{i}}^{a b} P_{L} \lambda_{r+A_{i}, r+A_{i}+A_{4}}^{b c} \Phi_{r+A_{i}+A_{4}, r}^{\dagger c a}-\bar{\Psi}_{r, r+A_{i}}^{a b} P_{L} \Phi_{r+A_{i}, r-A_{4}}^{\dagger b c} c_{r-A_{4}, r}^{c a}\right] \\
& -\sqrt{2} i g \epsilon_{i j k}\left[\bar{\Psi}_{r, r+A_{i}}^{a b} P_{L} \Psi_{r+A_{i}, r+A_{i}+A_{j}}^{b c} \Phi_{r-A_{k}-A_{4}, r}^{c a}-\bar{\Psi}_{r, r+A_{i}}^{a b} P_{L} \Phi_{r+A_{i}, r+A_{i}+A_{k}+A_{4}}^{b c} \Psi_{r-A_{j}, r}^{c a}\right] \\
& -g^{2}\left(\Phi_{r, r+a_{i}}^{a b} \Phi_{r+a_{i}, r}^{\dagger b c}-\Phi_{r, r-a_{i}}^{\dagger a b} \Phi_{r-a_{i}, r}^{b c}\right)\left(\Phi_{r, r+a_{j}}^{c c} \Phi_{r+a_{j}, r}^{\dagger d a}-\Phi_{r, r-a_{j}}^{\dagger c d} \Phi_{r-a_{j}, r}^{d a}\right) \\
& +4 g^{2}\left(\Phi_{r, r+a_{i}}^{a b} \Phi_{r+a_{i}, r+a_{i}+a_{j}}^{b c} \Phi_{r+a_{i}+a_{j}, r+a_{j}}^{\dagger c d} \Phi_{r+a_{j}, r}^{\dagger d a}-\Phi_{r, r+a_{i}}^{a b} \Phi_{r+a_{i}, r+a_{i}+a_{j}}^{b c} \Phi_{r+a_{i}+a_{j}, r+a_{i}}^{\dagger c d} \Phi_{r+a_{i}, r}^{\dagger d a}\right)
\end{aligned}
$$

where $\mu, \nu=0,1,2,3$ are lorentz indices; $a, b, c, d=1$ to $N$ are $U(N)^{p}$ group labels; $r=1$ to $p$ labels the node of the quiver diagram (when the two node subscripts are equal it is an adjoint plus singlet and the two superscripts are in the same $\mathrm{U}(\mathrm{N})$ : when the two node subscripts are unequal it is a bifundamental and the two superscript labels transform under different $\mathrm{U}(\mathrm{N})$ groups); $a_{i} \quad(i=\{1,2,3\})$ label the first three of the 6 of $\mathrm{SU}(4) ; A_{m} \quad(m=\{1,2,3,4\})=$ $\left(A_{i}, A_{4}\right)$ label the 4 of $\mathrm{SU}(4)$. By definition $A_{4}$ denotes an arbitrarily-chosen fermion $(\lambda)$ associated with the gauge boson, similarly to the notation in the $\mathcal{N}=1$ supersymmetric case. Recall that $\sum_{m=1}^{m=4} A_{m}=0(\bmod \mathrm{p})$.

As we showed in the previous section, the infinite sequence of nonsupersymmetric $Z_{p}$ models can have scalars in adjoints (corresponding to $\left.a_{i}=0\right)$ and bifundamentals $\left(a_{i} \neq 0\right)$. Denoting by $x$ the number of the three $a_{i}$ which are non-zero, the models with $x=3$ have only bifundamental scalars, those with $x=0$ have only adjoints while $x=1,2$ models contain both types of scalar representations. As we have seen, to contain the phenomenologicallydesirable chiral fermions, it is necessary and sufficient that $x=3$.

Let us first consider the quadratic divergence question in the mother $\mathcal{N}=4$ theory. The $\mathcal{N}=4$ lagrangian is like Eq.(4) but since there is only one node all those subscripts become unnecessary so the form is simply

$$
\mathcal{L}=-\frac{1}{4} F_{\mu \nu}^{a b} F_{\mu \nu}^{b a}+i \bar{\lambda}^{a b} \gamma^{\mu} D_{\mu} \lambda^{b a}+2 D_{\mu} \Phi_{i}^{a b \dagger} D_{\mu} \Phi_{i}^{b a}+i \bar{\Psi}_{m}^{a b} \gamma^{\mu} D_{\mu} \Psi_{m}^{b a}
$$




$$
\begin{aligned}
& -2 i g\left[\bar{\Psi}_{i}^{a b} P_{L} \lambda^{b c} \Phi_{i, r}^{\dagger c a}-\bar{\Psi}_{i}^{a b} P_{L} \Phi_{i}^{b c} \lambda^{c a}\right] \\
& -\sqrt{2} i g \epsilon_{i j k}\left[\bar{\Psi}_{i}^{a b} P_{L} \Psi_{j}^{b c} \Phi_{k}^{\dagger c a}-\bar{\Psi}_{i}^{a b} P_{L} \Phi_{j}^{b c} \Psi_{k}^{c a}\right] \\
& -g^{2}\left(\Phi_{i}^{a b} \Phi_{i}^{\dagger b c}-\Phi_{i}^{\dagger a b} \Phi_{i}^{b c}\right)\left(\Phi_{j}^{c d} \Phi_{j}^{\dagger d a}-\Phi_{j}^{\dagger c d} \Phi_{j}^{d a}\right) \\
& +4 g^{2}\left(\Phi_{i}^{a b} \Phi_{j}^{b c} \Phi_{i}^{\dagger c d} \Phi_{j}^{\dagger d a}-\Phi_{i}^{a b} \Phi_{j}^{b c} \Phi_{j}^{\dagger c d} \Phi_{i}^{\dagger d a}\right)
\end{aligned}
$$

All $\mathcal{N}=4$ scalars are in adjoints and the scalar propagator has one-loop quadratic divergences coming potentially from three scalar self-energy diagrams: (a) the gauge loop (one quartic vertex); (b) the fermion loop (two trilinear vertices); and (c) the scalar loop (one quartic vertex).

For $\mathcal{N}=4$ the respective contributions of (a, b, c) are computable from Eq.(5) as proportional to $g^{2} N(1,-4,3)$ which cancel exactly.

The $\mathcal{N}=0$ results for the scalar self-energies (a, b, c) are computable from the lagrangian of Eq.(4). Fortunately, the calculation was already done in [11]. The result is amazing! The quadratic divergences cancel if and only if $\mathrm{x}=3$, exactly the same "if and only if" as to have chiral fermions. It is pleasing that one can independently confirm the results of [11] directly from the interactions in Eq.(4) To give just one explicit example, in the contributions to diagram (c) from the last term in Eq.(4), the 1/N corrections arise from a contraction of $\Phi$ with $\Phi^{\dagger}$ when all the four color superscripts are distinct and there is consequently no sum over color in the loop. For this case, examination of the node subscripts then confirms proportionality to the kronecker delta, $\delta_{0, a_{i}}$. If and only if all $a_{i} \neq 0$, all the other terms in Eq.(4) do not lead to $1 / \mathrm{N}$ corrections to the $\mathcal{N}=4$.

Some comments on the literature are necessary. In the 1999 paper of Csaki, Skiba and Terning [12] it was claimed that there are always $1 / N$ corrections to spoil cancellation for finite $\mathrm{N}$ and that $N \geq 10^{28}$ is necessary! This was because of a technical error that the orbifolded gauge group is not $S U(N)^{p}$ but $U(N)^{p}$ and bifundamentals carry $\mathrm{U}(1)$ charges. A paper by Fuchs [13] in 2000, which has been largely ignored, corrected this point.

The conclusion is that the chiral $Z_{7}, Z_{12}$ models of $[8,9]$ which contain the standard model are free of one-loop quadratic divergences in the scalar propagator. Nevertheless the overall 
conformal invariance would not be respected by $U(1)$ factors which would have non-zero positive beta-functions. Clearly these factors must somehow be decoupled. This mysterious decoupling of U(1)'s from AdS/CFT which would not be conformally invariant has been

commented upon in $[14,15]$. A better understanding of these $\mathrm{U}(1)$ 's may be necessary to achieve the hope of a fully four-dimensionally conformally invariant extension of the standard model. There is the paradoxical requirement that the $\mathrm{U}(1)$ gauge factors must be present to cancel quadratic divergences but must decouple to preserve 4-dim conformal invariance at high energy.

Eventually gravity, at the Planck scale, will inevitably break conformal invariance because Newton's constant is dimensionful. A realistic hope is that there is a substantial window of energy scales where conformal invariance is an excellent approximation between, say, $4 \mathrm{TeV}$ [9] for at least a few orders of magnitude in energy even towards a scale approaching the see-saw scale $\sim 10^{10} \mathrm{GeV}$. It is difficult to foresee how large the conformality window is. Finally it is interesting to note that the present models seem to have all the ingredients of the so-called little Higgs models [16], which were proposed later than [6], with the quiver diagram here interpreted as the theory space there.

\section{ACKNOWLEDGEMENTS}

One of us (PHF) thanks D.R.T. Jones for discussions. This work was supported in part by the U.S. Department of Energy under Grant No. DE-FG02-97ER-41036 


\section{REFERENCES}

[1] I. Jack and D.R.T. Jones, Phys. Lett. B234, 321 (1990).

[2] S. Weinberg, Phys. Rev. D13, 974 (1975); ibid D19, 1277 (1979). L. Susskind, Phys. Rev. D20, 2619-2625 (1979); E. Farhi and L. Susskind, Phys. Reports, 74, 277 (1981).

[3] J. Wess and B. Zumino, Nucl. Phys. B70, 39-50 (1974); H.P. Nilles, Phys. Reports, 110, 1 (1984). H.E. Haber and G.L. Kane, Phys. Reports, 117, 75 (1985).

[4] I. Antoniadis, Phys. Lett. B246, 377-384 (1990). N. Arkani-Hamed, S. Dimopoulos and G.R. Dvali, Phys. Lett. B429, 263-272 (1998). hep-ph/9803315. I. Antoniadis, N. Arkani-Hamed, S. Dimopoulos and G.R. Dvali, Phys. Lett. B436, 257-263 (1998). hep-ph/9804398.

[5] W. Lerche, D. Lust and A.N. Schellekens, Nucl. Phys. B287, 477 (1987).

R. Bousso and J. Polchinski, JHEP 06: 006 (2000).

[6] P.H. Frampton, Phys. Rev. D60, 085004 (1999). hep-th/9812117.

[7] P.H. Frampton and C. Vafa. hep-th/9903226

[8] P.H. Frampton, Phys. Rev. D60, 121901 (1999). hep-th/9907051. see also: ibid 085004 (1999). hep-th/9905042.

[9] P.H. Frampton, Mod. Phys. Lett. A18, 1377 (2003). hep-ph/0208044. see also:

P.H. Frampton, R.M. Rohm and T. Takahashi, Phys. Lett. B570, 67 (2003). hep-ph/0302074.

[10] P.H. Frampton and T.W. Kephart, Int. J. Mod. Phys. A19, 593 (2004). hep-th/0308207.

[11] P. Brax, A. Falkowski, Z. Lalak and S. Pokorski, Phys. Lett. B538, 426-434 (2002).

[12] C. Csaki, W. Skiba and J. Terning, Phys. Rev. D61, 025019 (1999). 
In this paper, the orbifolded gauge group was assumed to be $S U(N)^{p}$ rather than $U(N)^{p}$ and hence bifundamentals which do have U(1) charges were incorrectly incorporated.

[13] E. Fuchs, JHEP 0010: 028 (2000). hep-th/0003235.

[14] E. Witten, Adv. Theor. Math. Phys. 2, 253-291 (1998). hep-ph/9802150. Footnote 7.

[15] J. Polchinski, Int. J. Math. Phys. A16, 707-718 (2001). hep-th/0011193. Footnote at end of Section 3.1.

[16] N. Arkani-Hamed, A.G. Cohen, T. Gregoire, E. Katz, A.E. Nelson and J.G. Wacker, JHEP 0208:021 (2002). hep-ph/0206020. 